\title{
The Initial Mass Function of Young Open Clusters in the Galaxy: A Preliminary Result
}

\author{
Beomdu Lim ${ }^{1}$, Hwankyung Sung ${ }^{2}$, Hyeonoh Hur ${ }^{2}$, \\ and Byeong-Gon Park ${ }^{1,3}$
}

\author{
${ }^{1}$ Korea Astronomy and Space Science Institute, 776 Daedeokdae-ro, Yuseong-gu, Daejeon \\ 305-348, Korea \\ email: bdlim1210@kasi.re.kr \\ ${ }^{2}$ Dept. of Astronomy \& Space Science, Sejong University, 209 Neungdong-ro, Gwangjin-gu, \\ Seoul 143-747, Korea \\ ${ }^{3}$ Korea University of Science and Technology, Gajeong-ro, Yuseong-gu, Daejeon 305-333, Korea
}

\begin{abstract}
The initial mass function (IMF) is an essential tool with which to study star formation processes. We have initiated the photometric survey of young open clusters in the Galaxy, from which the stellar IMFs are obtained in a homogeneous way. A total of 16 famous young open clusters have preferentially been studied up to now. These clusters have a wide range of surface densities $\left(\log \sigma=-1\right.$ to 3 [stars $\mathrm{pc}^{-2}$ ] for stars with mass larger than $\left.5 M_{\odot}\right)$ and cluster masses $\left(M_{\mathrm{cl}}=165\right.$ to $\left.50,000 M_{\odot}\right)$, and also are distributed in five different spiral arms in the Galaxy. It is possible to test the dependence of star formation processes on the global properties of individual clusters or environmental conditions. We present a preliminary result on the variation of the IMF in this paper.
\end{abstract}

Keywords. stars: luminosity function, mass function, open clusters and associations: general

\section{Introduction}

It is well recognized that the evolution of stars mainly depends on their initial mass, partly on the chemical composition. However what determines the stellar mass is poorly understood. The origin of the stellar mass spectrum is thought to be closely related to star formation processes. And therefore the initial mass function (IMF), the mass distribution of stars from a single star formation event, is a crucial diagnostic tool with which to understand star formation processes.

Although the IMF seems to be universal according to a number of previous studies (Bastian, Covey, \& Meyer 2010), many evidences for the variation of the IMF have been reported both from observational and theoretical studies (Lu et al. 2013; Marks et al. 2012; etc). One of main reasons for this debate may be caused by the use of inhomogeneous data. Thus, we focus on the results from a homogeneous imaging survey of young open clusters in the Galaxy ("Sejong Open cluster Survey" - Sung et al. 2013).

\section{Preliminary Results and Conclusions}

The young open clusters are useful objects in the study of the IMF because they are not only a coeval and co-spatial population but also an ecological indicator in the Galaxy. We investigated the IMF of 16 famous young open clusters by analyzing their Hertzsprung-Russell diagrams. The upper left-hand panel of Fig. 1 shows their IMF and its slope $\Gamma$ (the data from Lim 2014, references therein, and ongoing work).

Variations of the slope $\Gamma$ with respect to the surface density and total mass of the clusters are shown in the upper right and lower left-hand panels, respectively. The slope 

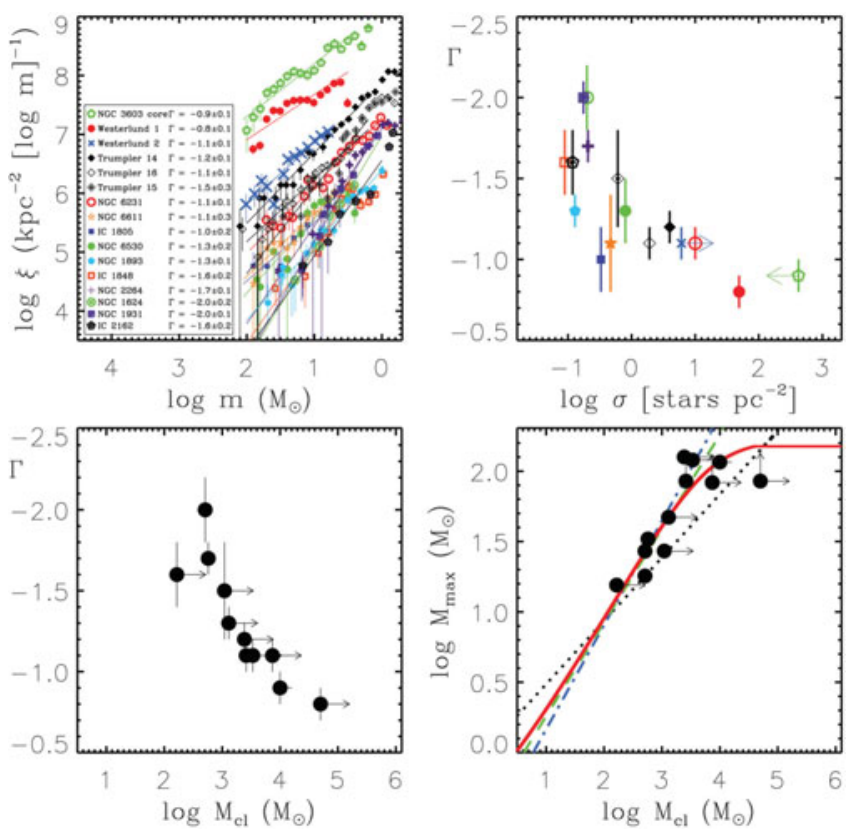

Figure 1. The initial mass functions (IMF) of 16 young open clusters in the Galaxy (upper left), variations of the IMF slope $\Gamma$ with respect to the surface density of the clusters (upper right) and the cluster mass (lower left), and a dependency of maximum stellar masses on the mass of host clusters (lower right). In the lower right-hand panel, the dot-dashed, dotted, dashed, and solid lines show $M_{\mathrm{max}}-M_{\mathrm{cl}}$ relations from analytic approaches of Elmegreen (2000), Larson (2003), Bonnell, Bate, \& Vine (2003), and Weidner \& Kroupa (2004), respectively.

of the IMF in the high-mass regime $\left(>1 M_{\odot}\right)$ appears to be shallow for massive dense clusters. The maximum stellar mass is also larger for more massive clusters (lower righthand panel). This $M_{\max }-M_{\mathrm{cl}}$ relation is consistent with results from analytic approaches (Elmegreen 2000; Larson 2003; Bonnell, Bate, \& Vine 2003; Weidner \& Kroupa 2004). It may imply the deterministic origin of the stellar mass.

The Galactic variations of the IMF and the cluster properties were also investigated to simply test the environmental influences on the star formation processes. The massive dense clusters tend to be formed in the inner Galaxy, while the formation of less massive sparse clusters seem to be dominant in the outer Galaxy. We tentatively suggest that the star formation is likely to be controlled by local environmental conditions rather than a universal process.

\section{References}

Bastian, N., Covey, K. R., \& Meyer, M. R. 2010, ARAA, 48, 339

Bonnell, A., Bate, M. R., \& Vine, S. G. 2003, MNRAS, 343, 413

Elmegreen, B. G. 2000, ApJ, 539, 342

Larson, R. 2003, ASP-CS Galactic Star Formation Across the Stellar Mass Spectrum, 287, 65

Lim, B. 2014, PhD thesis, Sejong Univ., Seoul, Korea

Lu, J. R., Do, T., Ghez, A. M., Morris, M. R., Yelda, S., \& Matthews, K. 2000, ApJ, 764, 155

Marks, M., Kroupa, P., Dabringhausen, J., \& Pawlowski, M. S. 2012, MNRAS, 422, 2246

Sung, H., Lim, B., Bessell, M. S., Kim, J. S., Hur, H., Chun, M.-Y., \& Park, B.-G. 2013, J. Korean Astron. Soc., 46, 103

Weidner, C. \& Kroupa, P. 2004, MNRAS, 348, 187 